\title{
Atomistic and Kinetic Simulations of Radiation Damage in Molybdenum
}

\author{
Zeke Insepov, ${ }^{1}$ Jeffrey Rest, ${ }^{1}$ Abdellatif M. Yacout, ${ }^{1}$ Bei Ye,${ }^{1}$ Di Yun, ${ }^{1}$ \\ Alexey Y Kuksin, ${ }^{2}$ Genri E Norman, ${ }^{2}$ Vladimir V Stegailov, ${ }^{2}$ Alexey V Yanilkin ${ }^{2}$ \\ ${ }^{1)}$ Argonne National Laboratory, Argonne, Illinois, USA \\ ${ }^{2)}$ Joint Institute for High Temperatures, Moscow, Russian Federation
}

\begin{abstract}
An ab initio quantum mechanics theory was applied to develop a new interatomic potential for Mo. The new potential was used to study formation and time evolution of radiation defects, such as self-interstitial atoms (SIAs), vacancies, and small clusters of SIAs, using molecular dynamics (MD). MD models were developed for calculation of the diffusion coefficients of vacancies, self-interstitials, and small dislocation loops containing 2 to 37 SIAs; and the rate constants were calculated. Interactions of small SIA loops with SIAs were simulated. The results show that rotation of SIA from one $<111>$ to another equivalent direction is an important mechanism that significantly contributes to kinetic coefficients.
\end{abstract}

\section{INTRODUCTION}

Evolution of self-interstitial atoms (SIAs) and vacancies is the first stage of relaxation of a damaged structure after an intense ion bombardment and radiation cascade in metallic fuels. This stage plays an important role in the nucleation processes of dislocations and voids. The modern kinetic theory of radiation damage describes the kinetics of these processes in terms of the rate constants and interaction radius [1].

Recent MD results [2-8] demonstrated high mobility of dislocation loops composed of SIAs. The mobility of the dislocation loops was also observed experimentally in [9]. The description of loop in the kinetic rate theory is based on the sink strength of the dislocation loop [10], in which the loop growth and shrinkage are determined by the diffusion of point defects (SIA, vacancies). A recent ion bombardment experiment conducted on Mo thin films demonstrated a high escaping rate of the dislocation loops during the irradiation [11].

For the work presented here, ab initio calculations were used to establish a new embedded atom model (EAM)-interatomic potential of pure Mo [12], which is then used for static and dynamic atomistic simulations of the rates of binary reactions between the defects in Mo: of SIA with SIA and with vacancy. This potential was parameterized by a force-matching method (FMM), with a large set of configurations of defects and therefore was capable of reproducing a correct potential energy map and a hierarchy of the formation and migration energies of the defects. The calculations were carried out with the LAMMPS code [13].

In this paper, the results of combination of this newly developed Mo interatomic potential 
with MD calculations of the diffusion coefficients of SIAs, vacancies, and dislocation loops containing 2 to 37 SIAs are presented, as well as the kinetic rate coefficients of dimer formation and defect recombination. The calculated data was supplied to a meso-scale kinetic rate theory model recently developed at Argonne National Laboratory as input parameters. The results from the rate theory model, such as dislocation loop size and number density were compared with experimental data [19].

\section{DIFFUSION COEFFICIENTS OF VACANCIES AND SELF-INTERSTITIALS IN MO}

An MD model was developed for studying evolution of point defects in a pure Mo system. The point defects were uniformly distributed in the system at the beginning of the simulations. A typical size of simulation cell for the simulations of SIA and SIA reactions was $100 \times 100 \times 100$ lattice units $(31 \times 31 \times 31 \mathrm{~nm})$. The simulated system contained about 2 million atoms. The initial numbers of SIAs were 300,400 , and 600 . For evaluation of the recombination rates between vacancy and SIA, a $100 \times 100 \times 100$ system with 200 SIAs and $800,1000,1500$, and 2000 vacancies, and a $150 \times 150 \times 150$ system with 200 SIAs and 3000, 4000, 5000, and 6000 vacancies were used. Throughout, 3-dimensional (3D) periodic boundary conditions were applied.

The rate constants of SIA clustering $\alpha_{\mathrm{ij}}$ and recombination rate constants $\alpha_{\mathrm{iv}}$ were calculated at different temperatures. Two different data sets were obtained that corresponded to two different analyses for each reaction of second order. This conclusion differs from that presented in $[3,4]$, in which the reaction is third order. An alternative approach, referred to as the "jump method," results in a second-order reaction in the case of 1-dimentional (1D) diffusion [14]. Experimental data on self-diffusion in pure Mo are given in Refs. $[15,16]$.

Our calculations have shown that a $<111>$ dumbbell has the lowest energy and a $<111>$ crowdion has almost the same energy and therefore these two types of defects are constantly transforming from one type to another. A $<110>$ dumbbell defect (a transient or rotation form) has a larger energy, $E_{\mathrm{a}, \mathrm{rot}}=E_{\mathrm{D} 110}-E_{\mathrm{D} 111}=0.194 \pm 0.03 \mathrm{eV}$ [17], and exists for a very short time. This defect contributes to $3 \mathrm{D}$ diffusion at higher temperatures as a closest saddle point for rotations between two equivalent $<111>$-directions.

In order to calculate the diffusion coefficients, point defects (vacancy or SIA) were created within the simulation box of size from $10 \times 10 \times 10$ to $30 \times 30 \times 30$ lattice constants containing a defect-free $b c c$ crystal under periodic boundary conditions. The diffusion coefficients of atoms (tracers diffusivity) due to defects were calculated from the mean-square-displacements (MSD) of the particles in the system. The self-diffusion coefficient due to each type of defects is related to the diffusivity of defects $D_{a}\left(a=v, i\right.$ denoting vacancy or SIA) by a correlation factor $f: D_{t}=f$ $D_{\alpha}$ [7]. The correlation factor $f$ can be evaluated theoretically for vacancies. The diffusion mechanism of interstitials is complex, and an analysis of the defect trajectory is required. The position of SIA, represented by $R(t)$, was determined as an atomic position with the highest potential energy and by taking into account the periodic boundary conditions. The diffusion coefficient was calculated in accordance with the formula: $D_{i}=\lim _{t \rightarrow \infty}\left\langle\bar{R}^{2}(t)\right\rangle / 6 t$. Averaging was

performed over a set of samplings for SIA migration over a long trajectory $(\sim 10 \mathrm{~ns})$. The calculated data can be fitted by an Arrhenius formula at low temperatures, where a corresponding activation energy is $0.02 \mathrm{eV}$ for SIA.

Figures 1 and 2 show the diffusion coefficients of vacancies, self-interstitials, and selfdiffusion coefficient calculated by MD in this work by using the expression [7] 


$$
D_{\text {self }}=f_{v} D_{v}+f_{\text {sia }} D_{\text {sia }},
$$

where correlation factors $f$ and the partial diffusion coefficients correspond to those of the vacancy and self-interstitial ones. All coefficients are presented in Figs. 1 and 2. Figure 2 shows a comparison of the MD calculated results with two existing experimental data sets [15, 16].
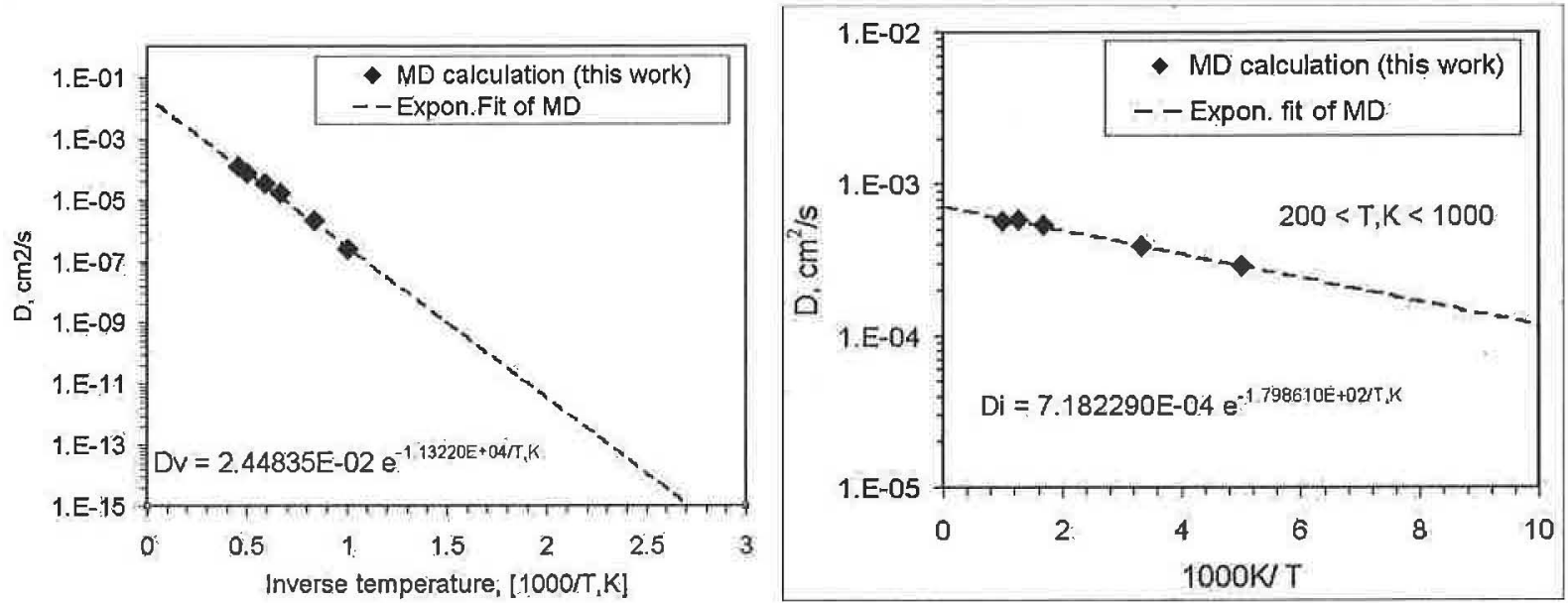

Figure 1. Diffusion coefficients of a) vacancies and b) interstitials of molybdenum calculated in this paper by MD using the new Mo-Xe potential developed at Argonne [12].

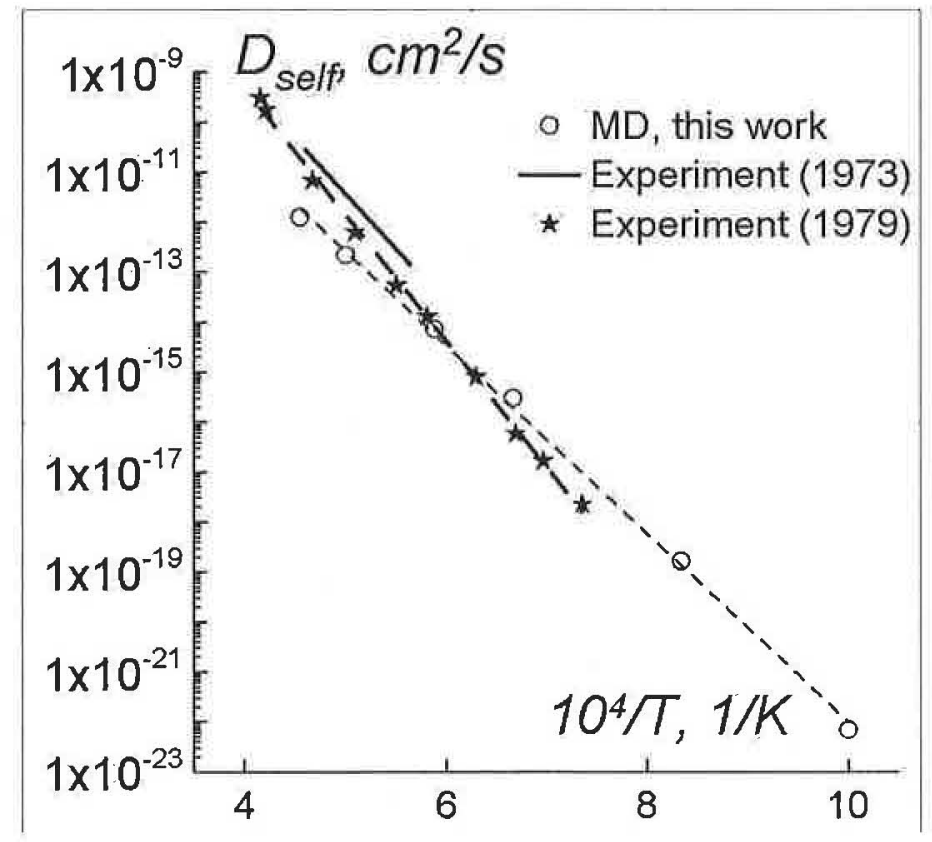

Figure 2. Self-interstitial diffusion coefficient of molybdenum calculated in this work by MD, can be approximated at low temperatures: The MD results are compared with two experimental data sets $[15,16]$.

The diffusion coefficient of vacancies calculated in this work was approximated by the expression $D_{\mathrm{v}}=2.44835 \times 10^{-2} \exp (-1.1322 \mathrm{e} 4 / T,[K])$, in $\mathrm{cm}^{2} / \mathrm{s}$. The diffusion coefficient of selfinterstitials was approximated at temperatures $T=200-1000 \mathrm{~K}$ as the expression: $D_{\mathrm{i}}=7.18229 \times$ 
$10^{-4} \exp (-1.79861 \mathrm{e} 2 / T,[K])$, in $\mathrm{cm}^{2} / \mathrm{s}$.

Figure 2 shows the self-diffusion coefficients of Mo calculated by MD in this work by using the expression in equation (1). [7] and comparison of our result with two existing experimental data sets $[15,16]$.

Additionally, MD calculations of the diffusion coefficients of dislocation loops, containing $n$ SIAs $(n=2-37)$, were carried out. 3D periodic boundary conditions were applied. The diffusion coefficients of dislocation loops were calculated via a time dependence of the SIA cluster meansquare-displacement as follows: $D_{\text {loop }}=M S D(t) / 2 t$. The calculated results were well fitted with the following expression: $D_{\mathrm{n}} \approx 2.3 \times 10^{-3} / \mathrm{n}^{-0.488}\left(\mathrm{~cm}^{2} / \mathrm{s}\right)$, where $n$ is the loop size (the number of SIAs in the loop).

\section{KINETIC COEFFICIENTS OF DIMER FORMATION AND RECOMBINATION}

\section{Dimer formation rate constant}

The SIA-dimer formation rate constant $\left(\alpha_{\mathrm{ii}}\right)$ was calculated by MD in our previous paper by two methods. The following two rate constants were obtained at $300 \mathrm{~K}: \alpha_{\mathrm{md}(1)}$ and $\alpha_{\mathrm{md}(2) \text {, which }}$ deviate by a factor of $4: \alpha_{\mathrm{MD}(1)}=4 \times 10^{13}(1 / \mathrm{s})$ for the jump method [17] and $\alpha_{\mathrm{MD}(2)}=1 \times 10^{13}(1 / \mathrm{s})$ for the diffusion method [18]; $\left\langle\alpha_{\mathrm{MD}}\right\rangle=2.5 \times 10^{13}(1 / \mathrm{s})$ is an average between these two values. Since the diffusion motion at low temperatures $(5700 \mathrm{~K})$ is fully one-dimensional, an additional factor proportional to exp $\left(-E_{\mathrm{rot}} / k_{\mathrm{B}} T\right)$ was added to the MD rate constants. This exponential factor takes into account the activation barrier for rotation for two SIAs form one dimer (di-SIA): $\alpha_{i i}=<\alpha_{M D}>\xi \xi \alpha_{0} \exp \left(-E_{i m} / k T\right)$ where $<\alpha_{M D}>=2.5 \times 10^{13}(1 / \mathrm{s}), \xi=1.0 \exp \left(-E_{\mathrm{rot}} / k T\right), E_{\mathrm{im}}=0.02$ $\mathrm{eV}$ is the migration energy for interstitials, $E_{\mathrm{rot}}=0.164 \mathrm{eV}$ is the rotation activation barrier, and $\alpha_{0}=2.1677$ is the normalization factor.

\section{Rate constant of SIA-vacancy recombination}

The rate constant of SIA recombination with a vacancy in Mo was calculated by MD in a previous paper [18]: $\alpha_{i v}=\alpha_{0}^{i v} \exp \left(-E_{a} / k_{B} T\right)$. The following values were accepted in this paper as reference MD data: $\alpha_{\mathrm{iv}}=2 \times 10^{12}(1 / \mathrm{s})$ at $300 \mathrm{~K}$.

\section{Rate constant for dislocation loop growth}

The rate constant for dislocation loop growth was obtained in this work by MD calculations of a Mo crystalline sample containing of about $2 \times 10^{6}$ Mo atoms and various number $(2,4,6$, and 8 ) of dislocation loops, each of them built by 37 self-interstitials, at $300 \mathrm{~K}$. The corrected kinetic

rate constant can be expressed as follows: $\alpha_{i L}=\xi 2 \pi Z_{i}(\rho) D_{i} \sqrt{N_{l} c c^{2} / \pi} / \Omega$, where the following constant was used: $\xi=1.0 \exp \left(-E_{\mathrm{rot}} / k_{\mathrm{B}} T\right)$. The diffusion coefficient of interstitials $D_{\mathrm{i}}=D_{0} \exp (-$ $\left.E_{\text {im }} / k_{\mathrm{B}} T\right)$, where $D_{0}=7.18 \times 10^{-4} \mathrm{~cm}^{2} / \mathrm{s}$, and $E_{\text {im }}=0.02 \pm 0.01 \mathrm{eV}, c c=1.692 \times 10^{-8} \mathrm{~cm}$. A similar probability was estimated for the loop-loop association rate constant.

\section{KINETIC THEORY OF DISLOCATION LOOP GROWTH}

The MD-calculated rate constants are applied in the kinetic equations of interstitial dislocation loop growth: 


$$
\begin{aligned}
& \frac{d c_{i}}{d t}=K-\alpha_{i v} c_{i} c_{v}-\alpha_{i i} c_{i} c_{i}-\alpha_{i L} c_{i} N_{L}, \\
& \frac{d c_{v}}{d t}=K-\alpha_{i v} c_{i} c_{v}-\alpha_{v L} c_{v} N_{L}, \\
& \frac{d N_{l}}{d t}=\frac{1}{2} \alpha_{i i} c_{i} c_{i} / \Omega, \\
& \frac{d m_{L}}{d t}=\alpha_{i L} c_{i}-\alpha_{v L} c_{v},
\end{aligned}
$$

where $K$ is the damage rate, $c_{i}, c_{v}$, and $N_{L}$ are the average SIA, vacancy, and interstitial loop density, respectively; $m_{L}$ is the average number of atoms in the average-size loop; and $\alpha_{i v}, \alpha_{i i}$, $\alpha_{i L}$, and $\alpha_{v L}$ are the kinetic coefficients (rate constants) in terms of binary reactions. The gain and loss of irradiation generated defects and impurities are tracked by various terms in the constitutive equations, each term being associated with an assumed behavioral mechanism. The analytic form of the terms representing the various physical processes is generally derived based on a continuum approach from mean field theory.

The rate theory model is then used in a proof-of-concept exercise to interpret the evolution of dislocation loops in electron-irradiated molybdenum. Calculations of the dose dependence of average loop density and diameter were performed and compared with experimental measurements obtained from irradiations with 1-2 MeV electrons. Based on the qualitative agreement between key loop growth characteristics and the experimental data, we conclude that the kinetic rate theory model is able to reasonably describe defect evolution during electron irradiation at room temperature in the absence of significant surface effects. Detailed validation process and results can be found in [19].

\section{CONCLUSIONS}

A multi-scale concept is proposed that consists of using ab-initio and atomistic simulation methods, verified with experimental data, in order to validate the mean-field theory kinetic approach as a universal tool for the nuclear materials. This concept is based on kinetic rateequations for radiation damage, energetics and kinetics of defects. Since the interaction potentials are important for the new concept, they were developed based on a force-matching method using data from ab initio calculations or were fitted to existing experimental data. These potentials were then used to study defect mobility, accumulation, and diffusion. SIA+SIA and vacancy+SIA reactions were studied by MD simulations, which play a crucial role in the initial stages of radiation damage. The influence of $1 \mathrm{D}$ diffusion on kinetics was studied. Although the diffusion of SIA is strongly one-dimensional at $T=300 \mathrm{~K}$, the reactions of SIA+SIA is described by three dimensional diffusion at high temperatures. The same conclusion is drawn for the recombination of vacancies and SIAs.

The transition from the sessile configuration to the mobile one of SIA-dimers was also studied. The activation energy for the transition was obtained.The rate constants of SIA+SIA and vacancy+SIA reactions were calculated based on the kinetics, and the temperature dependences were obtained.

They were implemented in a kinetic rate theory model with the intent of reducing the inherent uncertainty in predicting material behavior under irradiation. This multi-scale modeling approach 
links the atomistic scale calculations, which extract information in the nanometer and nanosecond scales, with mesoscale calculations that focus on microstructure evolution.

\section{ACKNOWLEDGMENTS}

This work was supported by the U.S. Department of Energy, under Contract DE-AC02-06CH11357.

\section{REFERENCES}

[1] J. Rest, J. Nucl. Mat. 277, 231(2000).

[2] B.D. Wirth, G.R. Odette, D. Maroudas, G.E. Lucas, J. Nucl. Mat. 276, 33 (2000).

[3] J. Marian, B. D. Wirth, A. Caro, B. Sadigh, G. R. Odette, J. M. Perlado, and T. Diaz de la Rubia, Phys Rev B 65, 144102 (2002).

[4] Yu. N. Osetsky; D. J. Bacon; A. Serra; B. N. Singh; S. I. Golubov, Phil. Mag. 83, 61 (2003).

[5] D. A. Terentyev, L. Malerba, and M. Hou, Phys. Rev. B 75, 104108 (2007).

[6] K. Arakawa, K. Ono, M. Isshiki, K. Mimura, M. Uchikoshi, H. Mori, Science 318, 956 (2007).

[7] N. Soneda, T. Diaz de La Rubia, Phil. Mag. A 81:2, 331 (2001).

[8] Yu. N. Osetsky, D.J. Bacon, A. Serra, B.N. Singh, S.I. Golubov, J. Nucl. Mater. 276, 65 (2000).

[9] Y. Matsukawa, S. J. Zinkle, Science 318, 959 (2007).

[10] A. D. Brailsford, R. Bullough, Phil. Trans. R. Soc. London A 302, 87 (1981).

[11] D. Yun, G. L. Hofman, Y. S. Kim, A. M. Yacout, M. Stan, Finite Element Modeling of Irradiation Induced Swelling and Creep in Metallic Mini-plate Fuel - A Preliminary Study, Trans. Am. Nucl. Soc.; 2011 American Nuclear Society (ANS) Winter Meeting and Nuclear Technology Expo; Washington D.C. ;Oct. 30, 2011 - Nov. 3, 201

[12] S. V. Starikov, Z. Insepov, J. Rest, A. Yu. Kuksin, G. E. Norman, V. V. Stegailov, A. V. Yanilkin, Phys. Rev. B 84, 104109 (2011).

[13] S. J. Plimpton: J. Comp. Phys. 117, 1 (1995).

[14] G.V. Kidson: J. Nucl. Mat. 118,115 (1983).

[15] K. Maier, H. Mehrer, and G. Rein, Z.f.Metallk., 70, 271 (1979).

[16] G.B. Federov, E.A. Smirnov, V.N. Gusev, F.I. Zhomov and V.L. Gorbenko, in Metallurgy and Materials Science of Pure Metals, Atomizdat, Moscow, 10, 62 (1973).

[17] A. Yanilkin, Z. Insepov, G. Norman, J. Rest, V. Stegailov, Proc. of DIMAT Conf. (2011).

[18] Z. Insepov, A.Yu. Kuksin, J. Rest, S. Starikov, A. Yanilkin, A.M. Yacout, B. Ye, D. Yun, arXiv:1203.0327v1 [cond-mat.mtrl-sci] (2012).

[19] B.Ye, D. Yun, Z. Insepov, J. Rest, presented at the 2012 MRS Spring Meeting, San Francisco, CA, 2012 (unpublished).

Government License

The submitted manuscript has been created by UChicago Argonne, LLC, Operator of Argonne National Laboratory ("Argonne"). Argonne, a U.S. Department of Energy Office of Science laboratory, is operated under Contract No. DE-AC02-06CH11357. The U.S. Government retains for itself, and others acting on its behalf, a paid-up nonexclusive, irrevocable worldwide license in said article to reproduce, prepare derivative works, distribute copies to the public, and perform publicly and display publicly, by or on behalf of the Government. 